\title{
A Prominent Role of PDIA6 in Processing of Misfolded Proinsulin
}

Dhana G Gorasia ${ }^{1}$, Nadine L Dudek ${ }^{1,2}$, Helena Safavi-Hemami ${ }^{1, \# a}$, Rochelle Ayala Perez $^{2}$, Ralf B Schittenhelm ${ }^{2}$, Philippa M Saunders ${ }^{3}$, Sheena Wee ${ }^{1 \# b}$, Jon E Mangum ${ }^{4}$, Michael J Hubbard ${ }^{5}$, Anthony W Purcell ${ }^{1,2} *$

Keywords: Proinsulin, PDI, chaperones, protein folding, disulfide bonds, ERAD 


\begin{abstract}
Despite its critical role in maintaining glucose homeostasis, surprisingly little is known about proinsulin folding in the endoplasmic reticulum. In this study we aimed to understand the chaperones involved in the maturation and degradation of proinsulin. We generated pancreatic beta cell lines expressing FLAG-tagged proinsulin. Several chaperones (including BiP, PDIA6, calnexin, calreticulin, GRP170, Erdj3 and ribophorin II) co-immunoprecipitated with proinsulin suggesting a role for these proteins in folding. To investigate the chaperones responsible for targeting misfolded proinsulin for degradation, we also created a beta cell line expressing FLAG-tagged proinsulin carrying the Akita mutation (Cys96Tyr). All chaperones found to be associated with wild type proinsulin also co-immunoprecipitated with Akita proinsulin. However, one additional protein, namely $\mathrm{P} 58^{\mathrm{IPK}}$, specifically precipitated with Akita proinsulin and approximately ten fold more PDIA6, but not other PDI family members, was bound to Akita proinsulin. The latter suggests that PDIA6 may act as a key reductase and target misfolded proinsulin to the ER-degradation pathway. The preferential association of PDIA6 to Akita proinsulin was also confirmed in another beta cell line ( $\beta \mathrm{TC}-6)$. Furthermore, for the first time, a physiologically relevant substrate for PDIA6 has been evidenced. Thus, this study has identified several chaperones/foldases that associated with wild type proinsulin and has also provided a comprehensive interactome for Akita misfolded proinsulin.
\end{abstract}




\section{Introduction}

The insulin molecule is synthesised as preproinsulin and translocated to the endoplasmic reticulum (ER), where immediately after translocation the signal peptide is cleaved by a signal peptidase to generate proinsulin [1]. Proinsulin contains three disulfide bonds; two disulfides linking the $\mathrm{A}$ and $\mathrm{B}$ chains of insulin and an additional intrachain disulfide bond within the $\mathrm{A}$ chain [2]. A number of ER-resident chaperones/folding enzymes, such as protein disulfide isomerase (PDI), are thought to assist in the folding of proinsulin to ensure the correct formation of disulfide bonds prior to its translocation from the ER to the Golgi apparatus [3, 4]. In the trans-Golgi, proinsulin is assembled into proinsulin hexamers in the presence of zinc ions, and packaged into vesicles that bud from the trans-Golgi network [1, 2]. Within the secretory granules proteolytic cleavage of the proinsulin hexamers by proconvertases and carboxypeptidases at the B chain/C-peptide junction and the A chain/C-peptide junction leads to the formation of insulin hexamers and C-peptides that are released into the circulation upon glucose stimulation $[1,5]$.

In addition to its important role in controlling glucose homeostasis, insulin is a significant autoantigen in type 1 diabetes (T1D), however it is unclear what triggers the autoreactive immune response to proinsulin [6-8]. Aberrant forms of proinsulin may be more immunogenic than the native hormone as it has been shown that CD4+ T cells isolated from T1D patients specifically recognize misfolded proinsulin [9, 10]. In addition, transgenic mice expressing mutant proinsulin (Akita mutation) where the cysteine at position 7 in the A chain, which normally engages in a crucial disulfide bond, is mutated to tyrosine develop diabetes $[11,12]$. In addition to this mutant form of proinsulin, Akita mice express wild type proinsulin from three alleles (two Ins1 and one Ins2), which should be more than sufficient to control glucose homeostasis and avoid diabetes. However, these mice experience beta cell loss a few weeks after birth and subsequently develop diabetes in absence of inflammation [12, 13].

Proteomics studies have shown that proteins thought to play a role in insulin biosynthesis (e.g PDI, ERO1L- $\beta$ ) are altered in beta cells when exposed to proinflammatory cytokines $[14,15]$. We hypothesised that changes in the abundance of 
these molecules may lead to accumulation of misfolded proinsulin that in turn leads to ER stress. Very little is known about chaperone-assisted proinsulin folding and misfolding. A recent study by Pottekot et al. characterised 230 proteins that interacted with proinsulin/insulin during its biosynthesis [16]. Of these 230 proteins, 21 were found to interact with proinsulin at early stages of synthesis and folding [16]. However, an interactome for misfolded proinsulin has yet to be determined. Of note, Hartley et al. [17], identified genes that were affected by the inducible expression of mutant proinsulin in a beta cell line, but did not focus on investigating the proteins that interact with mutant proinsulin.

Here we were interested in identifying the foldases and chaperones involved in proinsulin production (folding and misfolding). FLAG-tagged proinsulin and mutant Akita proinsulin were expressed in the NIT-1 pancreatic beta cell line. Importantly, the tags provided unique immunoreactivity with anti-FLAG-monoclonal antibodies, and so enabled specific immunoprecipitation of proinsulin and associated molecules, irrespective of the conformation or processing of the introduced insulin molecule. Moreover, FLAG-tagged Akita proinsulin was specifically immunoprecipitated using this approach avoiding precipitation of endogenous wild type insulin associated with the use of insulin-specific antibodies. We identified several chaperones and foldases that associated with both wild type and Akita proinsulin and also a few that specifically associated with the mutant proinsulin. We also found evidence of a stable association between protein disulfide isomerase A6 (PDIA6), a PDI family member, and proinsulin which has not been directly reported in the past.

\section{Experimental Methods}

\subsection{Tissue culture}

The SV40 transformed NIT-1 and $\beta$ TC-6 insulinoma cell lines $[18,19]$ were grown in DMEM (Life Technologies, Carlsbad, CA, USA) supplemented with $10 \%$ heat inactivated fetal calf serum (FCS) (Sigma-Aldrich, St Louis, MO, USA), 2 mM Lglutamine (MP Biomedicals, Santa Ana, CA, USA), 100 units/ml Benzyl-penicillin (CSL, Melbourne, Victoria, Australia), $0.1 \mathrm{mg} / \mathrm{ml}$ streptomycin sulphate (SigmaAldrich), $0.05 \mathrm{mM} \beta$-mercaptoethanol (Sigma-Aldrich), $5 \mathrm{mM}$ HEPES buffer (MP 
Biomedicals) and $0.1 \mathrm{mM}$ non-essential amino acids (Life Technologies) herein referred to as DM-10 at $37^{\circ} \mathrm{C}$ and $10 \% \mathrm{CO}_{2}$. Transfected NIT-1 and $\beta$ TC-6 cell lines were cultured in DM-10 supplemented with geneticin $(0.5 \mathrm{mg} / \mathrm{ml})$ (Life Technologies) to maintain the expression of the transfected gene.

\subsection{Cloning and expression of FLAG-tagged proinsulin in NIT-1}

Murine preproinsulin tagged with FLAG-at either $\mathrm{C}$ or $\mathrm{N}$ terminus was cloned into the pcDNA3.1(-) vector. In brief, proinsulin was PCR amplified using primers containing the FLAG-tag. Primers used are listed in Table 1. The amplified PCR products were ligated into $p c D N A 3.1(-)$ vector and the integrity of the sequences was verified by nucleotide sequencing. These constructs were used to transfect NIT-1 cells using Lipofectamine 2000 (Life Technologies). Selected clones were matched for expression of the transfected protein by western blot analysis.

\section{Table 1: List of primers}

\begin{tabular}{cl}
\hline FLAG-C-terminal & \\
Forward: & 5'CGCGCTAGCCCAGCCTATCTTCCAGGTTA \\
Reverse: & 5'GCCGAATTCCTACTTGTCATCGTCGTCCTTGTAGTCGTTGCAGTAGTTCTCCAGCT \\
FLAG-N-terminal & \\
Forward: & 5'CGCCTCGAGGACTACAAGGACGACGATGACAAGTTTGTCAAGCAGCACCTTTG \\
Reverse: & 5' GCCGAATTCGTGGGTCTAGTTGCAGTAG \\
Signal seq & \\
Forward: & 5'CGCGCTAGCCCAGCCTATCTTCCAGGTTA \\
Reverse: & 5' CGCCTCGAGAGCCTGGGTGGGGTGGA \\
& \\
Akita Mutation & \\
Forward: & ATTGTAGATCAGTGCTACACCAGCATCTGCTCC \\
Reverse: & GGAGCAGATGCTGGTGTAGCACTGATCTACAAT \\
\hline
\end{tabular}

\subsection{Site directed mutagenesis, Akita proinsulin}

Constructs encoding FLAG-tagged Akita mutant proinsulin, in which cysteine at position 96 in the A chain was mutated to tyrosine, were generated using the Quik change site-Directed Mutagenesis kit (Agilent Stratagene, Santa Clara, California, USA). In brief, $125 \mathrm{ng}$ forward and reverse primer were added to PCR together with Pfu Turbo DNA polymerase and double stranded wild type FLAG-tagged proinsulin constructs as the template. Following PCR, DpnI was added to the tubes and 
transformed into XL1-Blue cells. The ampicillin resistant colonies containing the mutagenized construct were selected and verified by nucleotide sequencing.

\subsection{Cloning and expression of FLAG-tagged wild type and Akita proinsulin in $\beta$ TC-6}

Flag tagged murine preproinsulin and Akita preproinsulin were cloned into the PIRES2Zsgreen1 vector. Briefly, the plasmids $p c D N A 3.1(-)$ containing the FLAGtagged wild type and Akita proinsulin were digested with NheI and EcoRI to release the inserts and were then ligated into PIRES2Zsgreen1 vector. These constructs were used to transfect $\beta$ TC- 6 cells using lipofectamine 2000 . The transfectants were sorted to select for GFP positive cells and western blot analysis was performed to verify the expression of the transfected protein.

\subsection{Immunoblotting}

Cells were lysed at a density of $4 \times 10^{7}$ cells $/ \mathrm{ml}$ in lysis buffer containing $50 \mathrm{mM}$ Tris $(\mathrm{pH}$ 7.4), $150 \mathrm{mM} \mathrm{NaCl}, 0.5 \%$ Triton X-100 (LabChem, Zelienople, PA, USA) and complete protease inhibitors cocktail (Roche Applied Science, Castle Hill, NSW, Australia). Supernatants containing material from $2 \times 10^{5}$ cells were separated on $12 \%$ SDS-PAGE gels (Life Technologies) and the proteins transferred onto a nitrocellulose membrane (GE Healthcare, Little Chalfont, Buckinghamshire, United Kingdom) in the presence of $20 \mathrm{mM}$ Tris, $0.15 \mathrm{M}$ glycine and 20\% methanol. Membranes were blocked in 5\% skim milk in phosphate buffered saline with $0.05 \%$ Tween (PBST) washed in PBST prior to probing with primary and secondary antibodies. Primary antibodies: Anti-FLAG-(Sigma-Aldrich) 1:1000, Anti- $\beta$ Actin (Abcam, Cambridge, United Kingdom) 1:4000, Anti-PDIA6 (Abcam) 1:1000, Anti-PDI (produced in house [20]) 1:10000, Anti-Calnexin (produced in house) 1:500. Proteins were visualized using Western Lighting Chemiluminescence (Perkin Elmer, Waltham, MA, USA) using LAS3000 imaging system (FUJI, Japan). The bands on the membrane were quantitated using Image Quant (GE healthcare).

\subsection{Fluorescent Microscopy}


Cells were plated onto sterile Poly-L-lysine coated cover-slips at a density which would achieve a final confluence of $40-50 \%$ after overnight incubation. Cell were washed several times with PBS then fixed with $4 \%$ paraformaldehyde (Sigma-Aldrich). Following fixation, cells were permeabilised by treatment with $0.1 \%$ Triton $\mathrm{X}-100$ stained with anti-FLAG-antibody. A secondary FITC conjugated anti-mouse IgG antibody was used to visualize the FLAG-tagged proteins. The nuclear stain, Hoechst, was co-incubated with the cells, the cover-slips mounted with the anti-quenching agent MOWIOL (Calbiochem, Gibbstown, New Jersey, USA) onto glass slides (76x22mm) and allowed to settle for 1 hour. Stained cells were analysed on an upright Olympus Bx60 fluorescence microscope (Olympus, Notting Hill, Victoria, Australia).

\subsection{Co-immunoprecipitation of FLAG-tagged Proinsulin}

Transfected NIT-1 cells with FLAG-tagged proinsulin and parental NIT-1 cells were lysed for 30 minutes under non-reducing conditions on ice in TBS containing 1\% digitonin and complete protease inhibitor cocktail. The lysate was then centrifuged, precleared and immunoprecipitated using anti-FLAG-agarose (Sigma-Aldrich). The FLAG-agarose was then washed extensively in cold TBS containing $0.1 \%$ digitonin; once with cold solution containing $0.1 \%$ digitonin, $450 \mathrm{mM} \mathrm{NaCl}$ and $30 \mathrm{mM}$ Tris and a final wash in $10 \mathrm{mM}$ Tris, $\mathrm{pH}$ 7.4. The FLAG-agarose was transferred into a fresh Eppendorf tube before adding SDS loading buffer and boiling for 5 minutes. Half of the supernatant was directly loaded onto a 12\% SDS-PAGE gel and the other half was transferred into an Eppendorf tube containing 100 mM DTT. The samples were boiled again and loaded onto the gel. The gels were stained with Coomassie colloidal blue stain. The co-immunoprecipitation studies in NIT-1 cell lines was performed at least 3 times and statistical significance was based on paired t-Test. Co-immunoprecipitation studies on $\beta$ TC- 6 cell lines were essentially performed as above except n-Dodecyl $\beta$-Dmaltoside (DDM) was utilised to lyse the cells.

\subsection{In-gel digestion, mass spectrometry and protein identification}

Protein bands were excised manually and washed with double distilled water and $100 \%$ acetonitrile (ACN) (Burdick and Jackson, Morristown, NJ, USA). The protein bands were reduced with $20 \mathrm{mM}$ DTT for 1 hour at $58^{\circ} \mathrm{C}$ followed by alkylation with $100 \mathrm{mM}$ 
iodoacetamide for 30 minutes in the dark. The bands were then destained in $50 \%$ ACN/50 mM TEAB (triethylammonium bicarbonate) (Sigma-Aldrich) followed by dehydration with $100 \%$ ACN. In-gel digestion was performed using sequencing-grade trypsin (Sigma-Aldrich) at a final concentration of $10 \mathrm{ng} / \mu \mathrm{L}$ in $25 \mathrm{mM}$ TEAB. The tryptic fragments extracted after overnight digestion were separated on a C18 reversedphase column (ProteCol nano column, $300 \AA$ and $3 \mu \mathrm{m}$, dimensions: $75 \mu \mathrm{m}$ x $100 \mathrm{~mm}$, SGE Analytical Sciences, Melbourne, VIC, Australia) and analysed using a Hybrid Quadrupole-TOF LC/MS/MS mass spectrometer (QSTAR Elite, AB SCIEX, Foster City, CA, USA). Solvent A contained $0.1 \%$ formic acid (Thermo Scientific, Waltham, MA, USA)/water and solvent B consisted of $95 \%$ ACN $/ 0.1 \%$ formic acid/water. Separation was performed with a solvent B gradient of 5-60 \% over 40 minutes. Data was acquired using Analyst QS software (version 2.0, AB SCIEX). Tryptic fragments were also analysed using Liquid Chromatography- tandem Mass Spectrometry (LCMS/MS) on an Agilent 1100 nano LC linked to an Agilent 1100 ion trap XCT plus mass spectrometer fitted with an Agilent HPLC-Chip CUBE source (Agilent Technologies, Santa Clara, CA, USA). The resulting peptide mass maps and the associated fragmentation spectra were collectively used to interrogate sequences present in SwissProt to generate statistically significant candidate identifications using Mascot (version 2.2, Matrix Science, Boston, USA) with the following settings: trypsin, 1 missed cleavage, carbamidomethyl-Cys as a fixed and oxidation of methionine as a variable modification, 1.2 Da precursor tolerance, 0.8 Da peptide fragment, error tolerant search included.

\section{Results}

\subsection{Generation of cell lines expressing FLAG-tagged proinsulin}

The constructs containing murine preproinsulin with a FLAG-tag engineered at either the $\mathrm{C}$ or $\mathrm{N}$ terminus of the proinsulin molecule were transfected into NIT-1 cells. The transfectants were screened for the expression of FLAG-tagged proinsulin by western blot and stably transfected clonal cell lines were established (Figure 1A and B). Clone 6 of FLAG-C-terminal Proinsulin and clone 1 of FLAG-N-terminal proinsulin were chosen for further experiments as they expressed high levels of tagged proinsulin. 
A

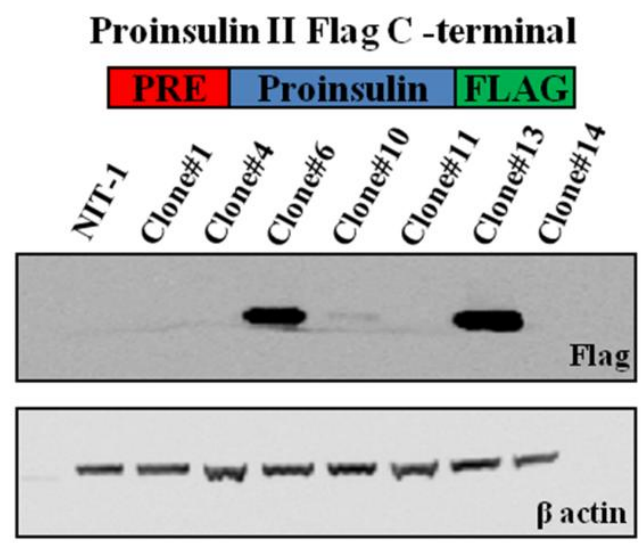

B

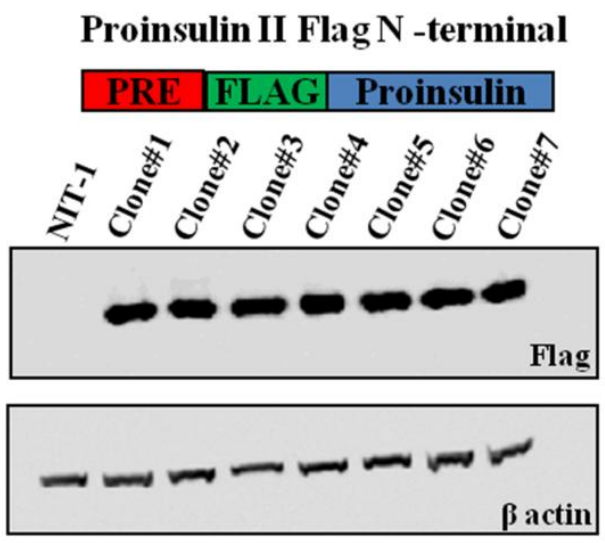

Figure 1: Cloning and expression of FLAG-tagged proinsulin in NIT-1 cells

Proinsulin cDNA was amplified by PCR using proinsulin specific primers and was cloned into pcDNA3.1(-) vector using NheI and EcoRI restriction sites. The FLAG-tag was added either on the C- or N-terminus of proinsulin. NIT-1 cells were transfected with the proinsulin constructs and individual clones were screened for proinsulin expression using anti-FLAG-antibody (A) FLAG-C-terminal proinsulin \& (B) FLAG$\mathrm{N}$-terminal proinsulin. Membranes were reprobed with anti-beta actin antibody as a loading control.

To determine whether the tagged proinsulin was folded properly and processed to insulin, the cells were lysed in 0.5\% CHAPS and tagged molecules immunoprecipitated. The eluted tagged proinsulin was analysed by SDS-PAGE under both reducing and nonreducing conditions. The theoretical molecular weights of proinsulin and insulin are 9.5 $\mathrm{kDa}$ and $5.7 \mathrm{kDa}$, respectively. The insulin separates into A $(2.4 \mathrm{kDa})$ and $\mathrm{B}(3.4 \mathrm{kDa})$ chains in the presence of DTT. Under non-reducing conditions FLAG-tagged proinsulin migrated at approximately $11 \mathrm{kDa}$ with a second band at approximately $5 \mathrm{kDa}$ only observed for C-terminal tagged insulin construct (Figure 2A). This second lower molecular weight band changed mobility under reducing conditions and migrated with an apparent molecular mass of approximately $3 \mathrm{kDa}$ (Figure $2 \mathrm{~A}$ ). These bands were excised, subjected to in-gel tryptic digestion and the extracted tryptic fragments were analysed by MS. The $\sim 5 \mathrm{kDa}$ band was identified as insulin (the B chain peptide, 
K.QHLCGSHLVEALYLVCGER.G) with a Mascot score of 102, an expect value of 2e-008 and percentage sequence coverage of $37 \%$. The $3 \mathrm{kDa}$ band was not identified by MS, however it most likely represents the FLAG-tagged A chain of insulin based on its migration pattern. Overall, these results suggest that only the C-terminally tagged proinsulin is processed to the mature hormone, a finding consistent with previous observations [21, 22]. Since C-terminally FLAG-tagged proinsulin was folded and processed properly, further studies were performed on cells expressing this construct. Next we investigated the localisation of the FLAG-tagged proinsulin by immunofluorescence microscopy. FLAG-tagged proinsulin was primarily localized next to the nucleus, and the punctuate appearance suggests that it was properly translocated into the ER (Figure 2B).

A

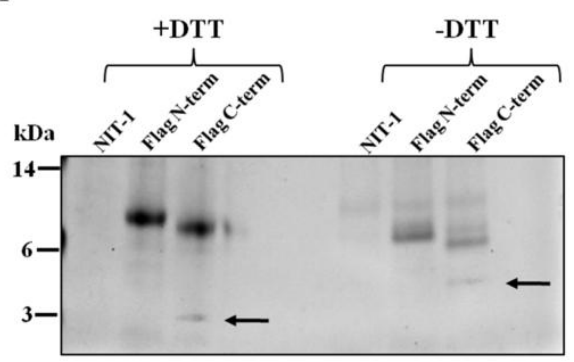

B

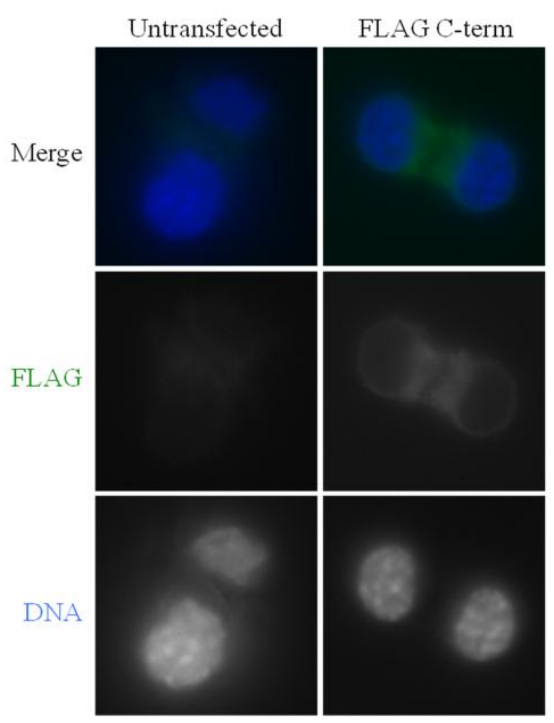

Figure 2: Processing and cellular localization of FLAG-tagged proinsulin in NIT-1 cells

(A) NIT-1 cells containing FLAG-tagged proinsulin were lysed in 0.5\% CHAPS and the FLAG-tagged proinsulin was pulled down using FLAG-agarose. The washed beads were resuspended in sample buffer and loaded onto a gel and stained with Coomassie blue. The arrows indicate mature FLAG-tagged insulin detected only in samples containing C-terminal tagged proinsulin. (B) Transfected NIT-1 cells were fixed and 
permeabilized on a coverslip and incubated with anti-FLAG-antibody followed by antimouse IgG FITC conjugate. The perinuclear pattern of FLAG-tagged proinsulin staining is consistent with its localisation in the ER.

\subsection{PDIA6 and other chaperones associated with wild type proinsulin}

There are at least two classes of proteins known to be involved in ER-mediated folding processes: enzymes that catalyze specific oxidation and isomerization steps and chaperones that stabilize unfolded or partially folded structures to prevent the formation of non-native intra- or interchain interactions [23]. In order to characterize these two classes of helper proteins, co-immunoprecipitation assays were performed. NIT-1 cells with and without tagged proinsulin were lysed in $1 \%$ digitonin, a mild non-ionic detergent that allows protein-protein interactions to be maintained. Tagged proinsulin and associated molecules were immunoprecipitated with FLAG-agarose then separated by SDS-PAGE under reducing and non-reducing conditions (Figure 3). As expected, the FLAG-tagged proinsulin bands, identified by MS, were only present in the transfected NIT-1 cells and the gel also revealed other proteins that specifically coimmunoprecipitated with proinsulin. The bands indicated with arrows were identified by MS (Table 2). Substantial quantities of immunoglobulin heavy chain binding protein (BiP) associated with tagged proinsulin (Figure 3, band 7). The reciprocal immunoprecipitation was also performed where BiP antibody was used to pull down proinsulin containing complexes followed by a western blot with FLAG-antibody, and a band reactive to anti-FLAG was observed (data not shown). DnaJ homolog subfamily B member 11/ ERdj3, a mammalian ER DnaJ cochaperone [24, 25], was also identified (Figure 3, band 5). A band co-migrating with ERdj3 was present in the negative control lane and using MS it was identified as actin, hence ERdj3 was considered a specific association. Other ER chaperones including calreticulin, calnexin, E3 ubiquitin-protein ligase (UBR2), Erlin-2 and glucose regulated protein 170 (GRP170) were specifically co-immunoprecipitated with proinsulin (Table 2). A glycoprotein-associated folding enzyme, ribophorin II which catalyses the transfer of a glycan to asparagine residues was also identified by co-immunoprecipitation (Figure 3, Table 2). In addition, two mitochondrial proteins, apoptosis-inducing factor mitochondrion-associated 1 and 
HSP60, co-precipitated with proinsulin. It is likely that these proteins bound artefactually, since HSP60 has been shown to bind opportunistically to protein complexes after cell lysis [26]. There are more than 20 members of the protein disulfide isomerase family (PDIs) present in the ER yet only a few are commonly abundant. As PDIA6 is not one of the latter [27], it was surprising that only PDIA6 (also known as P5/ERP5) (Figure 3, band 3, Table 2) was found to associate with tagged proinsulin. PDIA6 is an active oxidoreductase which has similar properties to other PDIs [28] and has been shown to be induced during the UPR [29, 30]. However, no previous studies have shown a direct involvement of PDIA6 in protein folding nor has a physiological substrate for PDIA6 been reported to our knowledge. The proteins that were observed to associate with FLAG-tagged proinsulin in here, except for PDIA6, have also been shown to interact with proinsulin in a study by Pottekat et al. [16].

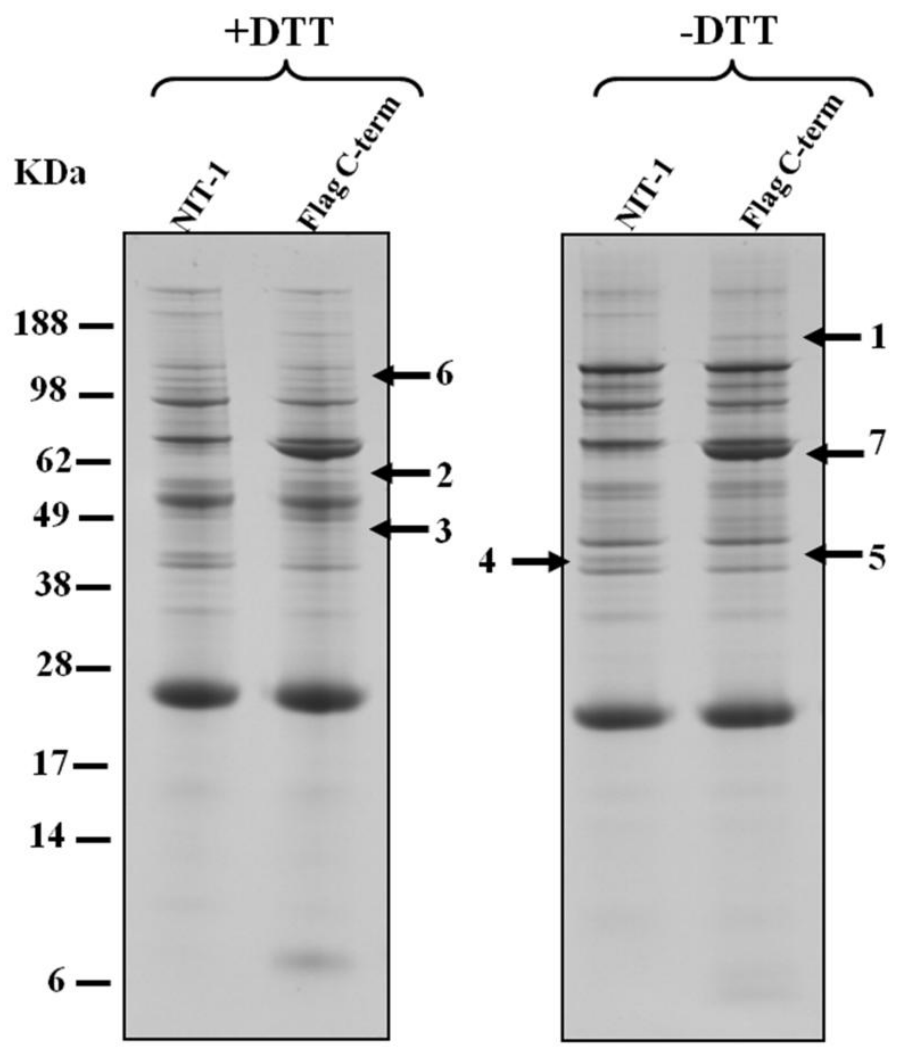

Figure 3: Proteins stably associated with wild type proinsulin in NIT-1 cells

(A) NIT-1 cells expressing FLAG-tagged proinsulin were lysed in 1\% digitonin and the FLAG-tagged proinsulin complexes were co-immunoprecipitated using FLAG-agarose. The washed beads were boiled in SDS to disrupt and elute the complex. The eluted 
samples were subjected to SDS-PAGE under reducing and non-reducing conditions and stained with Coomassie Blue. The bands that were only present in the FLAG-tagged proinsulin were considered specific associations and were excised and identified by MS. The corresponding bands were also excised from control lanes and analysed by MS to determine background proteins. See Table 2 for protein identifications. 
Table 2: Proteins that associate with wild type FLAG-tagged Proinsulin, identified by mass spectrometry

\begin{tabular}{|c|c|c|c|c|c|}
\hline Band & Protein Name & Accession No. & Mascot Score * & $\%$ seq Coverage & Peptide hits \\
\hline 1 & E3 ubiquitin-protein ligase UBR2 & Q6WKZ8 & 1995 & 25 & 33 \\
\hline \multirow[t]{3}{*}{2} & Apoptosis-inducing factor, mitochondrion & B1AU25 & 525 & 16 & 8 \\
\hline & Ribophorin II & A2ACG7 & 422 & 15 & 6 \\
\hline & $60 \mathrm{kDa}$ heat shock protein, mitochondrial (HSP60) & P63038 & 255 & 13 & 5 \\
\hline \multirow[t]{2}{*}{3} & Calreticulin & P14211 & 632 & 48 & 14 \\
\hline & Protein disulfide-isomerase A6, PDIA6 & Q922R8 & 311 & 12 & 3 \\
\hline 4 & Actin & P60710 & 1251 & 74 & 39 \\
\hline \multirow[t]{2}{*}{5} & DnaJ homolog subfamily B member 11, ERdj3 & Q99KV1 & 929 & 41 & 9 \\
\hline & Erlin-2 & Q8BFZ9 & 493 & 50 & 11 \\
\hline 6 & Glucose regulated protein $1(\mathrm{GRP} 170)$ & Q9JKR6 & 1106 & 28 & 21 \\
\hline \multirow[t]{3}{*}{7} & BiP/GRP78 & P20029 & 3294 & 65 & 37 \\
\hline & Heat shock cognate 71 (HSPA8) & P63017 & 1311 & 34 & 16 \\
\hline & Calnexin & P35564 & 256 & 15 & 6 \\
\hline
\end{tabular}

Note: *Mascot score greater than 37 is considered a significant hit $(\mathrm{p}<0.05)$ 


\subsection{Expression of FLAG-tagged Akita proinsulin in NIT-1 cells}

The Akita mutation (C96Y) was generated by site directed mutagenesis for FLAGtagged proinsulin. Clonal cell lines were established and screened by western blot (Figure 4A) and immunofluorescence microscopy, which suggested an ER localization (Figure 4B). Immunoprecipitation studies of tagged Akita proinsulin were performed and the associated proteins were separated by SDS-PAGE under reducing and nonreducing conditions. Distinct protein bands that were only present in the cell lines expressing the FLAG-tagged Akita proinsulin (Figure 5) were excised and identified by MS (Table 3). The analogous bands in the negative control lane were also analysed by MS to confirm specificity of association with mutant proinsulin.
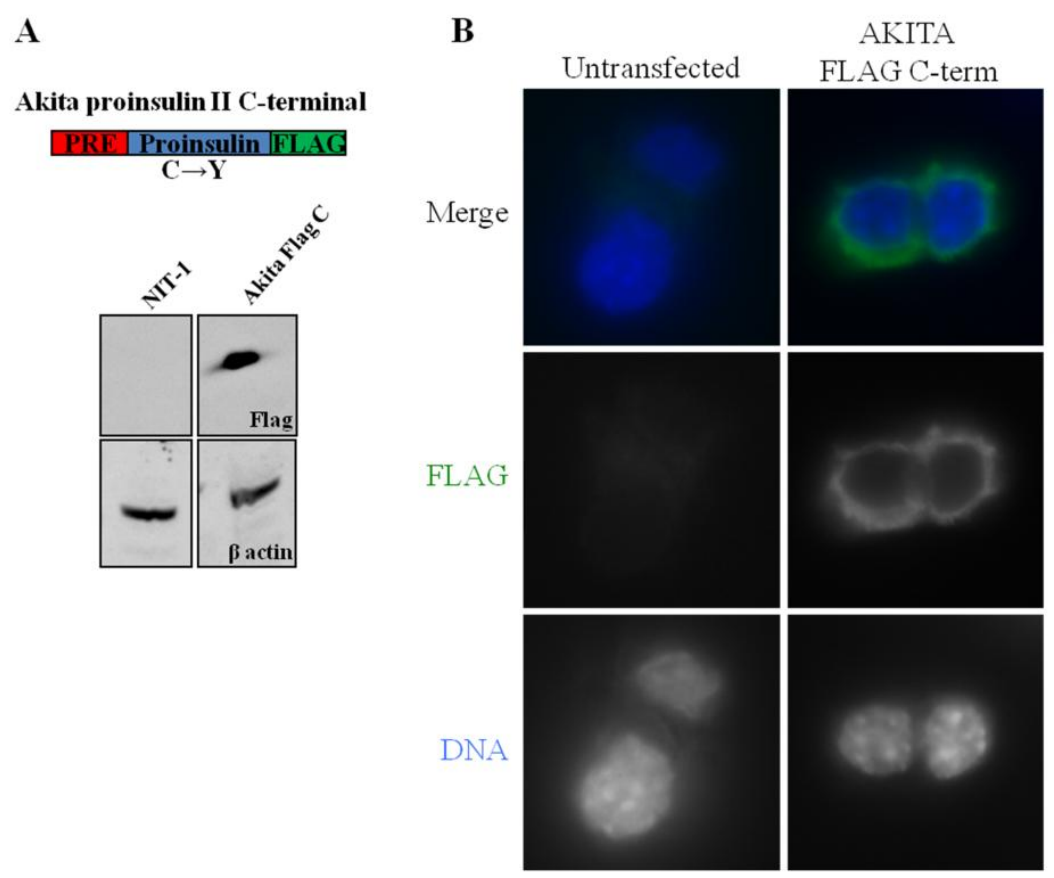

Figure 4: Expression of FLAG-tagged Akita proinsulin in NIT-1 cells and its subcellular localisation

NIT-1 cells were transfected with the Akita proinsulin constructs and the clones were screened for expression using anti-FLAG-antibody. (A) Western blot showing expression of C-terminal FLAG-tagged Akita proinsulin. The membrane was reprobed with anti- $\beta$ actin as a loading control. (B) Transfected NIT-1 cells were fixed and permeabilized on a coverslip and incubated with anti-FLAG-antibody followed by antimouse IgG FITC conjugate. The perinuclear pattern of FLAG-tagged proinsulin staining is consistent with ER localisation.

\subsection{Preferential association of PDIA6 with Akita proinsulin}


The majority of the proteins co-isolated with the Akita mutant proinsulin were also found associated with the wild type proinsulin (i.e. BiP, ERdj3, calreticulin, calnexin, erlin-2, apoptosis-inducing factor, Ribophorin II and HSP60). However, one heat shock protein 40 family member (DnaJ homolog subfamily C member 3/P58 ${ }^{\mathrm{IPK}}$ [31]) was only present in the complex associated with Akita proinsulin.

Importantly, even though PDIA6 associated with both wild type and Akita proinsulin, there was substantially more PDIA6 associated with the mutant proinsulin, based on the intensity of the co-precipitating band and semi-quantitative mass spectrometry (62 spectral counts versus 3 for PDIA6 bound to the mutant compared to wild type proinsulin respectively). To validate this finding immunoprecipitates from wild type and mutant proinsulin were immunoblotted using an antibody specific for PDIA6 plus a PDI isoform independent antibody (to evaluate the specific increase in the PDIA6 isoform). Approximately ten fold more PDIA6 was observed to associate with Akita compared to wild type proinsulin (Figure 6), confirming the MS results. 


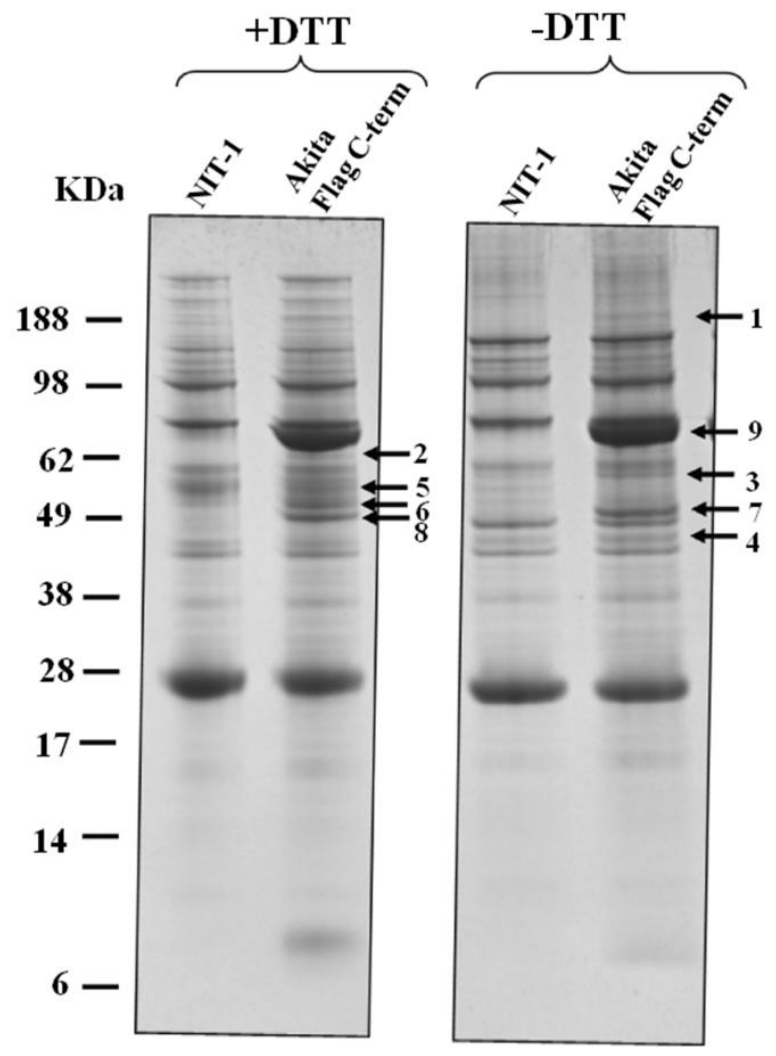

Figure 5: Proteins that associated with mutant FLAG-tagged Akita proinsulin in NIT-1 cells

NIT-1 cells expressing FLAG-tagged Akita proinsulin were lysed in 1\% digitonin and the FLAG-tagged Akita proinsulin complexes were co-immunoprecipitated using FLAG-agarose. The washed beads were boiled in SDS to elute the complex. The samples were subjected to SDS-PAGE under reducing and non-reducing conditions. Bands that were only present in the immunoprecipitates from the transfected cells were considered specific associations and were subsequently identified by Mass Spectrometry. The analogous bands in the control lane were also analysed by mass spectrometry to determine background protein. See Table 3 for protein identifications. 
Table 3: Proteins that associated with mutant proinsulin as identified by mass spectrometry

\begin{tabular}{|c|c|c|c|c|c|}
\hline Band & Protein Name & Accession No. & Mascot Score & \% seq Coverage & Peptide hits \\
\hline 1 & E3 ubiquitin-protein ligase UBR2 & Q6WKZ8 & 1246 & 17 & 27 \\
\hline \multirow[t]{3}{*}{2} & $60 \mathrm{kDa}$ heat shock protein, mitochondrial & P63038 & 902 & 36 & 16 \\
\hline & Apoptosis-inducing factor, mitochondrion & B1AU25 & 809 & 31 & 16 \\
\hline & Ribophorin II & A2ACG7 & 340 & 14 & 5 \\
\hline 3 & DnaJ homolog subfamily C member 3,P58 $8^{\mathrm{IPK}}$ & Q91YW3 & 1261 & 46 & 24 \\
\hline \multirow[t]{2}{*}{4} & DnaJ homolog subfamily B member 11,ERdj3 & Q99KV1 & 707 & 45 & 14 \\
\hline & Erlin-2 & Q8BFZ9 & 434 & 38 & 10 \\
\hline \multirow[t]{2}{*}{5} & Protein disulfide-isomerase A6 & Q922R8 & 809 & 35 & 13 \\
\hline & Calreticulin & P14211 & 245 & 10 & 5 \\
\hline $6,7,8$ & Protein disulfide-isomerase A6 & Q922R8 & 1375 & 72 & 62 \\
\hline \multirow[t]{3}{*}{9} & BiP/GRP78 & P20029 & 3518 & 86 & 140 \\
\hline & HSPA8 & P63017 & 1301 & 34 & 24 \\
\hline & Calnexin & P35564 & 269 & 16 & 6 \\
\hline
\end{tabular}


A

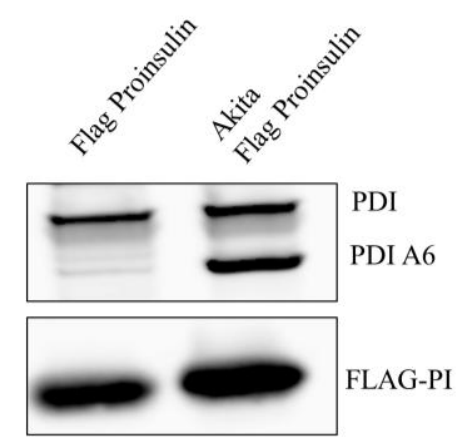

B

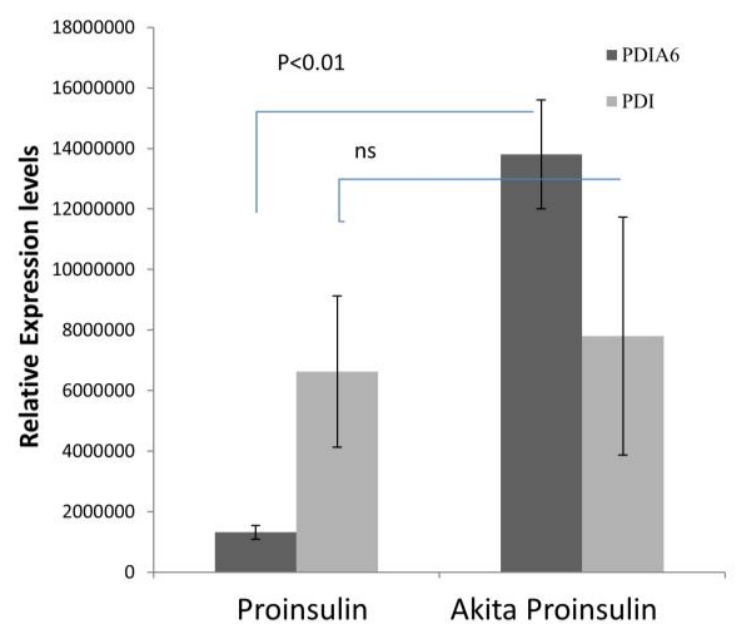

Figure 6: PDIA6 associates preferentially with mutant Akita proinsulin in NIT-1 cell line

NIT-1 cells expressing FLAG-tagged proinsulin or FLAG-tagged Akita proinsulin were lysed in $1 \%$ digitonin and the FLAG-tagged proinsulin and Akita proinsulin complexes were co-immunoprecipitated using FLAG-agarose. The eluted complex was separated on SDS-PAGE and immunoblotted for PDI, PDIA6 and FLAG. PDI and PDIA6 chemiluminescence was normalised to the total FLAG-immunoreactivity using fluorescence imaging. The data shown are from triplicate analyses and statistical significance is based on a paired t-Test.

To validate the preferential association of PDIA6 with mutant Akita proinsulin we generated another independent beta cell line, $\beta$ TC-6, expressing FLAG-tagged wild type and Akita proinsulin. Co-immunoprecipitation studies on these cell lines also showed increased association of PDIA6 with mutant Akita proinsulin which as anticipated failed to produce a mature insulin product (Figure 7). Additionally, we also identified PDI, calnexin and $\mathrm{BiP}$ association with both wild type and Akita proinsulin in this cell line (Figure 7). Collectively, this indicates that the data set obtained in NIT-1 cells does not only relate to this cell line and is perhaps reflective of what may happen in native proinsulin folding and targeting of misfolded proinsulin for degradation. 


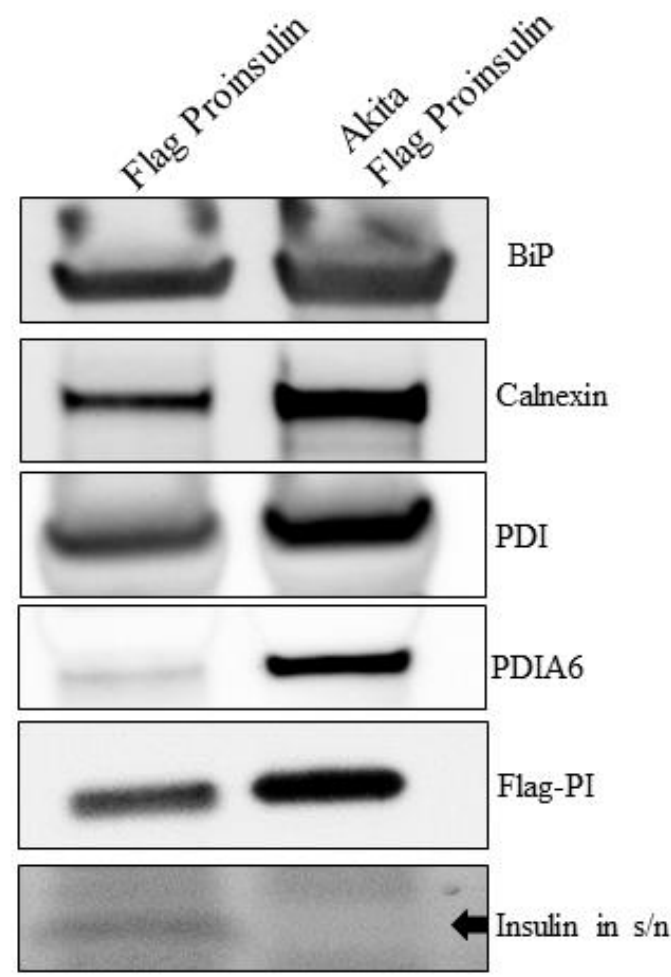

Figure 7: Association of PDIA6, PDI, BiP and calnexin with wild type and Akita proinsulin in $\beta$ TC- 6 cell line

Cells were lysed in 1\% DDM and the FLAG-tagged proinsulin and Akita proinsulin complexes were co-immunoprecipitated using FLAG-agarose. The eluted complexes were separated on SDS-PAGE and immunoblotted for PDIA6, PDI, BiP, calnexin and FLAG. Insulin secreted into the media was visualised by SDS-PAGE and Sypro ruby staining.

\section{Discussion}

Protein misfolding is increasingly being recognized as central to the progression of many diseases, including diabetes. The misfolding of proinsulin both in vitro and in vivo, involves predisposition to disulfide mispairing [32, 33]. Chaperones and folding enzymes play a crucial role both in ensuring the proper folding of proinsulin and the degradation of misfolded proinsulin. The goal of the present study was to examine the chaperones/folding enzymes involved in these processes. To identify ER chaperones that interact with wild type and mutant proinsulin, immunoprecipitations were performed on the NIT-1 cells expressing FLAG-tagged wildtype and mutant proinsulin.

In this work, the association of $\mathrm{BiP}$ with proinsulin was observed which has also been 
shown by previous studies investigating proinsulin folding [11, 34, 35]. BiP is an ER resident member of the HSP70 family and cooperates with co-chaperones of the DnaJ/HSP40 family that are responsible for stimulating the ATPase activity of BiP via their characteristic J-domains [25, 36]. BiP recognizes hydrophobic regions of peptides that are usually buried inside native proteins, and the binding and release of these unfolded or partially denatured substrates are ATP dependent [37]. Currently, seven mammalian ER DnaJ homologues have been identified and all of them have been shown in vitro to stimulate BiP ATPase activity [38] [39]. In this study ERdj3 was specifically identified as a putative co-chaperone for BiP during proinsulin folding. A previous study identified ERdj3 to interact with insulin during its biosynthesis [16]. ERdj3 is ubiquitously expressed, with highest levels of expression occurring in secretory tissues such as the pancreas [38]. It has also been shown by Shen et al. that ERdj3 associates with unfolded proteins that are BiP substrates [38].

Since proinsulin folding requires the formation of three disulfide bonds, one would anticipate that PDI plays a crucial role in the formation and rearrangement of the disulfide bonds. Previous studies have shown that PDI catalyses disulfide bond formation in proinsulin in vitro [3, 4]. Recently Rajpal et al. showed evidence of a direct interaction between PDI and proinsulin, however the authors suggested that PDI exhibits reductase activity and is involved in the retention of proinsulin in the ER [40]. Furthermore, studies where PDI was overexpressed [41] or knocked out [40] in beta cells did not enhance or impair disulfide bond formation in proinsulin. This suggests that there are other foldases involved in the disulfide editing of proinsulin. In this study, for the first time an association of proinsulin with a PDI family member, PDIA6, is shown. Like other PDI family members, PDIA6 has the two catalytic domains a and a' but lacks the two non-catalytic domains $b$ and b', required for substrate binding [42, 43]. Even though there are many PDIs that are localized in the ER, it was remarkable that proinsulin would stably associate with PDIA6 alone. Previously, it has been shown that PDIA6 reacts with substrates that are known to associate with BiP and that PDIA6 and BiP form a non-covalent complex [44]. However, more recently it was reported that knock out of PDIA6 did not have an effect on insulin secretion in the rat pancreatic beta cell line INS-1 [45]. Such lack of effect could reflect redundancy between PDIA6 other 
PDI isoforms for insulin folding, or that the proinsulin interactome differs between cell lines, (i.e a different member of PDI may be involved in proinsulin folding in INS-1 cells).

Interestingly, even though both wild type and mutant tagged proinsulin were expressed at similar levels in NIT-1 cells, substantially (10-fold) more PDIA6 associated with the Akita proinsulin. Previously, PDIA6 has been reported to interact with substrates indirectly via BiP [44] but recently a direct interaction of PDIA6 with IRE1 $\alpha$ has been shown [45]. The increase in co-precipitated PDIA6 may reflect a cysteine trapping effect of the Akita mutation, however, given that PDIA6 is a thiol-disulfide oxidoreductase and since Akita proinsulin is misfolded and therefore a substrate for ERAD, a likely role of PDIA6 in associating with Akita proinsulin could be to act as reductase to remove any disulfide bonds before retrotranslocation of Akita proinsulin from the ER to the cytosol. A similar role has been suggested for PDI [46] and ERdj5 [47]. Tsai et al. showed that PDI unfolds the A chain of cholera toxin in the ER before it is transported to the cytosol [46] and Ushioda et al. showed that ERdj5 accelerates the reduction and degradation of the NHK variant of $\alpha_{1}$-antitrypsin [47].

In addition to the increased levels of PDIA6, P58 ${ }^{\mathrm{IPK}}$ was found to specifically associate with the mutant but not wild type proinsulin. P58 ${ }^{\mathrm{IPK}}$ is an ER resident member of the dnaj family and its expression is induced by ER stress [29]. It is an important component of a negative feedback loop used by the cell to inhibit eIF- $2 \alpha$ signalling and attenuate the unfolded protein response (UPR) [48]. $\mathrm{P} 58^{\mathrm{IPK}}$ has been identified as cochaperone of $\mathrm{BiP}$, however, recent findings have indicated that $\mathrm{P} 58^{\mathrm{IPK}}$ makes a direct interaction with unfolded protein substrates, stabilises them, and then subsequently binds $\mathrm{BiP}$ and facilitates the transfer of the substrates to the chaperone $[49,50] . \mathrm{P}^{\mathrm{IPK}}$ knock out mice show a gradual loss of pancreatic beta cells and develop diabetes; due to sustained activation of the UPR and prolonged ER stress which induces apoptosis in beta cells [51]. P58 ${ }^{\mathrm{IPK}}$ is able to protect the stressed cells by not only attenuating the UPR but also by reducing the amount of proteins entering the already overloaded ER and thus giving a chance to the chaperones in the ER lumen to deal with the misfolded proteins [52]. In contrast, Rutkowski et al., showed that the mechanism of P58 ${ }^{\mathrm{IPK}}$ 
protection is due to the increased protein processing capacity in the ER [49], consistent with our observation that $\mathrm{P} 58^{\mathrm{IPK}}$ bound only to the mutant Akita proinsulin. Collectively, it appears that P58 ${ }^{\mathrm{IPK}}$ bound to the Akita mutant proinsulin and together with $\mathrm{BiP}$ it assisted in the degradation of Akita proinsulin and thus reducing the ER load.

Besides BiP, PDIA6 and P58 ${ }^{\mathrm{IPK}}$, calnexin, calreticulin and E3 ubiquitin-protein ligase were also shown to associate stably with both wild type and mutant proinsulin. Both calnexin and calreticulin are considered to be lectin chaperones since they bind to the monoglucosylated oligosaccharide present on N-linked glycoproteins [53]. Since proinsulin is not a glycoprotein it is unlikely that both calnexin and calreticulin are acting as lectin chaperones in this case. Indeed it has been demonstrated that calreticulin can bind directly to and chaperone the folding of the non-glycosylated proteins [54]. Experiments more directly relevant to chaperone function have shown that calreticulin and calnexin can bind and form stable complexes with unfolded, non-glycosylated proteins and suppress protein aggregation $[55,56]$. In the case of proinsulin folding, both calnexin and calreticulin may be acting as chaperones to prevent the aggregation of unfolded proinsulin or they could be binding to the other components of the folding complex. E3 ubiquitin-protein ligase or Hrd1 has been proposed to be involved in the retrotranslocation of the Akita proinsulin from the ER to the cytosol [57, 58], however, no direct interaction has been shown. In this study we show an association of both wild type and Akita proinsulin with E3 ubiquitin-protein ligase and confirm its role in the retrotranslocation of the misfolded proinsulin.

In summary, proinsulin production takes place in the ER with the help of a variety of molecular chaperones. In this study several novel associations of foldases/chaperones with both wild type and mutant proinsulin have been identified. Most importantly, previously no substrate of PDIA6 was known, and for the first time we show an enhanced association between mutant proinsulin and PDIA6 that may reflect a reductase role in the quality control of proinsulin biosynthesis. 


\section{Acknowledgments}

This work was supported by a Grant from the Juvenile Diabetes Research Foundation International (17-2012-134). AWP and SW acknowledge fellowship support from the Australian National Health and Medical Research Council. HSH is supported by a Marie Curie Fellowship from the European Union (CONBIOS 330486). MJH acknowledges Fellowship support from Melbourne Research Unit for Facial Disorders. RBS acknowledges Fellowship support from the Swiss National Science Foundation. 


\section{References}

1. Marques, R.G., M.J. Fontaine, and J. Rogers, C-peptide: much more than a byproduct of insulin biosynthesis. Pancreas, 2004. 29(3): p. 231-8.

2. Dodson, G. and D. Steiner, The role of assembly in insulin's biosynthesis. Curr Opin Struct Biol, 1998. 8(2): p. 189-94.

3. Tang, J.G., C.C. Wang, and C.L. Tsou, Formation of native insulin from the scrambled molecule by protein disulphide-isomerase. Biochem J, 1988. 255(2): p. 451-5.

4. Winter, J., et al., Catalytic activity and chaperone function of human proteindisulfide isomerase are required for the efficient refolding of proinsulin. J Biol Chem, 2002. 277(1): p. 310-7.

5. Dunn, M.F., Zinc-ligand interactions modulate assembly and stability of the insulin hexamer -- a review. Biometals, 2005. 18(4): p. 295-303.

6. Dubois-LaForgue, D., et al., T-cell response to proinsulin and insulin in type 1 and pretype 1 diabetes. J Clin Immunol, 1999. 19(2): p. 127-34.

7. Hassainya, Y., et al., Identification of naturally processed HLA-A2--restricted proinsulin epitopes by reverse immunology. Diabetes, 2005. 54(7): p. 2053-9.

8. Toma, A., et al., Recognition of a subregion of human proinsulin by class Irestricted T cells in type 1 diabetic patients. Proc Natl Acad Sci U S A, 2005. 102(30): p. 10581-6.

9. Mannering, S.I., et al., The insulin A-chain epitope recognized by human T cells is posttranslationally modified. J Exp Med, 2005. 202(9): p. 1191-7.

10. Mannering, S.I., et al., The A-chain of insulin is a hot-spot for CD4+ T cell epitopes in human type 1 diabetes. Clin Exp Immunol, 2009. 156(2): p. 226-31.

11. Wang, J., et al., A mutation in the insulin 2 gene induces diabetes with severe pancreatic beta-cell dysfunction in the Mody mouse. J Clin Invest, 1999. 103(1): p. 27-37.

12. Kayo, T. and A. Koizumi, Mapping of murine diabetogenic gene mody on chromosome 7 at D7Mit258 and its involvement in pancreatic islet and beta cell development during the perinatal period. J Clin Invest, 1998. 101(10): p. 21128.

13. Leroux, L., et al., Compensatory responses in mice carrying a null mutation for Ins1 or Ins2. Diabetes, 2001. 50 Suppl 1: p. S150-3.

14. D'Hertog, W., et al., Proteomics analysis of cytokine-induced dysfunction and death in insulin-producing INS-1E cells: new insights into the pathways involved. Mol Cell Proteomics, 2007. 6(12): p. 2180-99.

15. Gorasia, D.G., et al., Pancreatic beta cells are highly susceptible to oxidative and ER stress during the development of diabetes. J Proteome Res, 2014.

16. Pottekat, A., et al., Insulin biosynthetic interaction network component, TMEM24, facilitates insulin reserve pool release. Cell Rep, 2013. 4(5): p. 92130 .

17. Hartley, T., et al., Endoplasmic reticulum stress response in an INS-1 pancreatic beta-cell line with inducible expression of a folding-deficient proinsulin. BMC Cell Biol, 2010. 11: p. 59.

18. Hamaguchi, K., H.R. Gaskins, and E.H. Leiter, NIT-1, a pancreatic beta-cell line established from a transgenic NOD/Lt mouse. Diabetes, 1991. 40(7): p. 8429. 
19. Poitout, V., et al., Morphological and functional characterization of beta TC-6 cells--an insulin-secreting cell line derived from transgenic mice. Diabetes, 1995. 44(3): p. 306-13.

20. Shnyder, S.D., J.E. Mangum, and M.J. Hubbard, Triplex profiling of functionally distinct chaperones (ERp29/PDI/BiP) reveals marked heterogeneity of the endoplasmic reticulum proteome in cancer. J Proteome Res, 2008. 7(8): p. 336472.

21. Pouli, A.E., et al., Insulin targeting to the regulated secretory pathway after fusion with green fluorescent protein and firefly luciferase. Biochem J, 1998. 331 ( Pt 2): p. 669-75.

22. Ohara-Imaizumi, M., et al., Imaging exocytosis of single insulin secretory granules with evanescent wave microscopy: distinct behavior of granule motion in biphasic insulin release. J Biol Chem, 2002. 277(6): p. 3805-8.

23. Gething, M.J. and J. Sambrook, Protein folding in the cell. Nature, 1992. 355(6355): p. 33-45.

24. Bies, C., et al., A Scjlp homolog and folding catalysts present in dog pancreas microsomes. Biol Chem, 1999. 380(10): p. 1175-82.

25. Yu, M., R.H. Haslam, and D.B. Haslam, HEDJ, an Hsp40 co-chaperone localized to the endoplasmic reticulum of human cells. J Biol Chem, 2000. 275(32): p. 24984-92.

26. Meunier, L., et al., A subset of chaperones and folding enzymes form multiprotein complexes in endoplasmic reticulum to bind nascent proteins. Mol Biol Cell, 2002. 13(12): p. 4456-69.

27. Hatahet, F. and L.W. Ruddock, Protein disulfide isomerase: a critical evaluation of its function in disulfide bond formation. Antioxid Redox Signal, 2009. 11(11): p. 2807-50.

28. Alanen, H.I., et al., $p H$ dependence of the peptide thiol-disulfide oxidase activity of six members of the human protein disulfide isomerase family. Antioxid Redox Signal, 2006. 8(3-4): p. 283-91.

29. Lee, A.H., N.N. Iwakoshi, and L.H. Glimcher, XBP-1 regulates a subset of endoplasmic reticulum resident chaperone genes in the unfolded protein response. Mol Cell Biol, 2003. 23(21): p. 7448-59.

30. Belmont, P.J., et al., Roles for endoplasmic reticulum-associated degradation and the novel endoplasmic reticulum stress response gene Derlin-3 in the ischemic heart. Circ Res, 2010. 106(2): p. 307-16.

31. Melville, M.W., et al., The cellular inhibitor of the PKR protein kinase, P58(IPK), is an influenza virus-activated co-chaperone that modulates heat shock protein 70 activity. J Biol Chem, 1999. 274(6): p. 3797-803.

32. Min, C.Y., Z.S. Qiao, and Y.M. Feng, Unfolding of human proinsulin. Intermediates and possible role of its C-peptide in folding/unfolding. Eur $\mathrm{J}$ Biochem, 2004. 271(9): p. 1737-47.

33. Liu, M., et al., Proinsulin disulfide maturation and misfolding in the endoplasmic reticulum. J Biol Chem, 2005. 280(14): p. 13209-12.

34. Liu, M., et al., Proinsulin maturation, misfolding, and proteotoxicity. Proc Natl Acad Sci U S A, 2007. 104(40): p. 15841-6.

35. Schmitz, A., et al., In vivo iodination of a misfolded proinsulin reveals colocalized signals for Bip binding and for degradation in the ER. EMBO J, 1995. 14(6): p. 1091-8. 
36. Greene, M.K., K. Maskos, and S.J. Landry, Role of the J-domain in the cooperation of Hsp40 with Hsp70. Proc Natl Acad Sci U S A, 1998. 95(11): p. 6108-13.

37. Bukau, B. and A.L. Horwich, The Hsp70 and Hsp60 chaperone machines. Cell, 1998. 92(3): p. 351-66.

38. Shen, Y. and L.M. Hendershot, ERdj3, a stress-inducible endoplasmic reticulum DnaJ homologue, serves as a cofactor for BiP's interactions with unfolded substrates. Mol Biol Cell, 2005. 16(1): p. 40-50.

39. Otero, J.H., B. Lizak, and L.M. Hendershot, Life and death of a BiP substrate. Semin Cell Dev Biol, 2010. 21(5): p. 472-8.

40. Rajpal, G., et al., Action of protein disulfide isomerase on proinsulin exit from endoplasmic reticulum of pancreatic beta-cells. J Biol Chem, 2012. 287(1): p. 43-7.

41. Zhang, L., et al., GRP78, but Not Protein-disulfide Isomerase, Partially Reverses Hyperglycemia-induced Inhibition of Insulin Synthesis and Secretion in Pancreatic \{beta\}-Cells. J Biol Chem, 2009. 284(8): p. 5289-98.

42. Ellgaard, L. and L.W. Ruddock, The human protein disulphide isomerase family: substrate interactions and functional properties. EMBO Rep, 2005. 6(1): p. 28-32.

43. Klappa, $\mathrm{P}$., et al., The $b^{\prime}$ domain provides the principal peptide-binding site of protein disulfide isomerase but all domains contribute to binding of misfolded proteins. EMBO J, 1998. 17(4): p. 927-35.

44. Jessop, C.E., et al., Protein disulphide isomerase family members show distinct substrate specificity: P5 is targeted to BiP client proteins. J Cell Sci, 2009. 122(Pt 23): p. 4287-95.

45. Eletto, D., et al., Protein disulfide isomerase A6 controls the decay of IRE1alpha signaling via disulfide-dependent association. Mol Cell, 2014. 53(4): p. 562-76.

46. Tsai, B., et al., Protein disulfide isomerase acts as a redox-dependent chaperone to unfold cholera toxin. Cell, 2001. 104(6): p. 937-48.

47. Ushioda, R., et al., ERdj5 is required as a disulfide reductase for degradation of misfolded proteins in the ER. Science, 2008. 321(5888): p. 569-72.

48. van Huizen, R., et al., P58IPK, a novel endoplasmic reticulum stress-inducible protein and potential negative regulator of eIF2alpha signaling. $\mathrm{J}$ Biol Chem, 2003. 278(18): p. 15558-64.

49. Rutkowski, D.T., et al., The role of p58IPK in protecting the stressed endoplasmic reticulum. Mol Biol Cell, 2007. 18(9): p. 3681-91.

50. Tao, J., et al., Crystal structure of P58(IPK) TPR fragment reveals the mechanism for its molecular chaperone activity in UPR. J Mol Biol, 2010. 397(5): p. 1307-15.

51. Ladiges, W.C., et al., Pancreatic beta-cell failure and diabetes in mice with a deletion mutation of the endoplasmic reticulum molecular chaperone gene P58IPK. Diabetes, 2005. 54(4): p. 1074-81.

52. Oyadomari, S., et al., Cotranslocational degradation protects the stressed endoplasmic reticulum from protein overload. Cell, 2006. 126(4): p. 727-39.

53. Williams, D.B., Beyond lectins: the calnexin/calreticulin chaperone system of the endoplasmic reticulum. J Cell Sci, 2006. 119(Pt 4): p. 615-23.

54. Jorgensen, C.S., et al., Polypeptide binding properties of the chaperone calreticulin. Eur J Biochem, 2000. 267(10): p. 2945-54. 
55. Ihara, Y., et al., Calnexin discriminates between protein conformational states and functions as a molecular chaperone in vitro. Mol Cell, 1999. 4(3): p. 33141.

56. Saito, Y., et al., Calreticulin functions in vitro as a molecular chaperone for both glycosylated and non-glycosylated proteins. EMBO J, 1999. 18(23): p. 6718-29.

57. He, K., et al., PDI reductase acts on Akita mutant proinsulin to initiate retrotranslocation along the Hrdl/Sel1L-p97 axis. Mol Biol Cell, 2015. 26(19): p. 3413-23.

58. Hoelen, H., et al., Proteasomal Degradation of Proinsulin Requires Derlin-2, HRD1 and p97. PLoS One, 2015. 10(6): p. e0128206. 


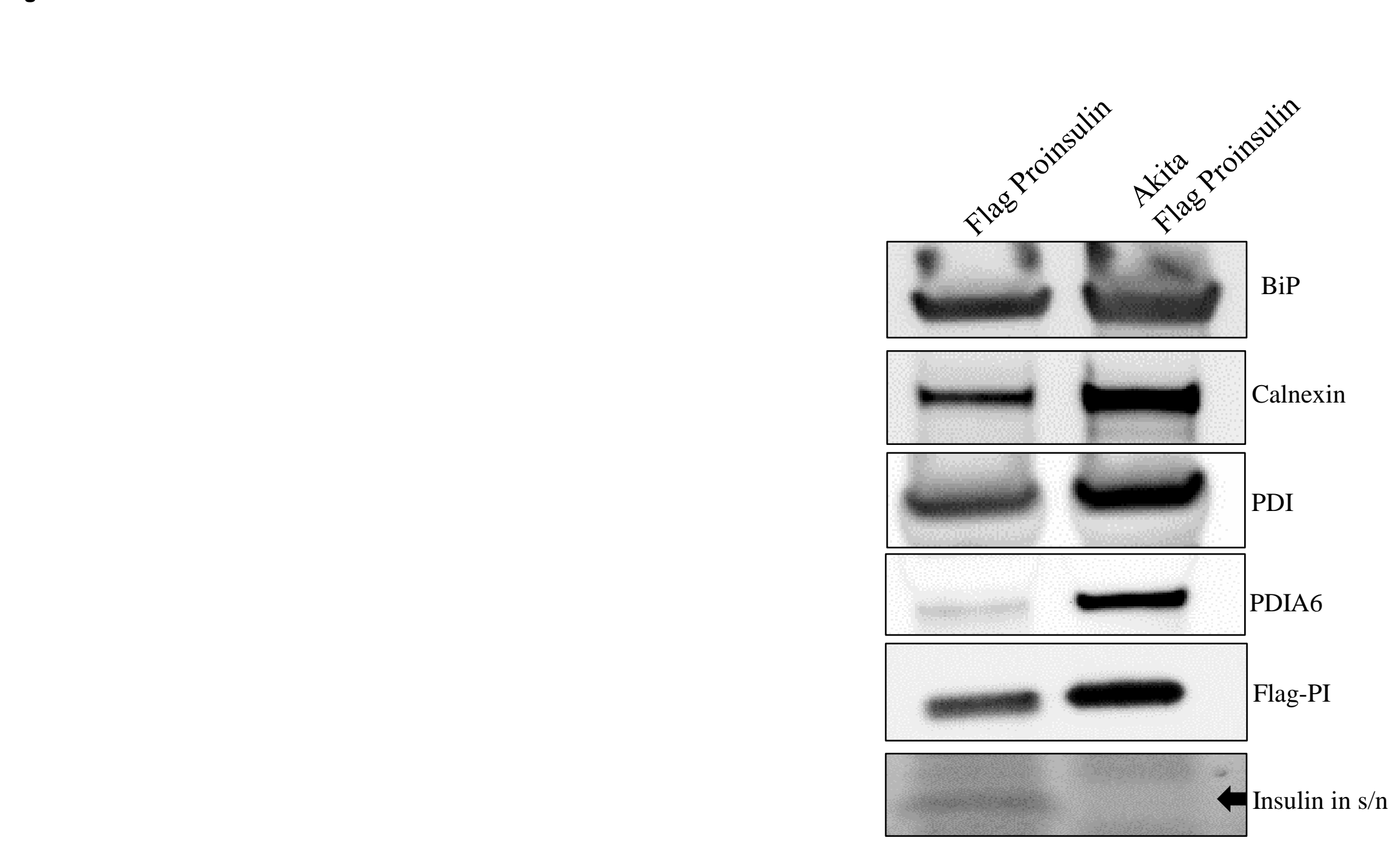

